\section{A BASIC program package for the analysis of sorting data}

\author{
THOMAS ECKES \\ Universität des Saarlandes, Saarbrücken, \\ Federal Republic of Germany
}

The sorting method has proved to be a valuable datagathering procedure in many research areas (see, e.g., Rosenberg, 1982). Subjects are typically asked to sort a given set of objects into as many groups or classes as they wish so that objects within the same class are more similar to each other than they are to objects in other classes. If the classes are mutually exclusive and collectively exhaustive, the subjects' sortings are called partitions (see Boorman \& Arabie, 1972). Compared with related program abstracts (Grant, 1983; Greenberg \& McIsaac, 1984; Oud \& Sattler, 1984; Takane, 1981, 1982; Weldon \& Buchter, 1984), the present program package is distinctive in that it performs (1) a two-way analysis of sorting data (i.e., computation of proximities between objects as well as computation of proximities between partitions), (2) a significance test of the proximities between objects, and (3) an assessment of correspondence between partitions by means of two measures differing in the kind of weighting scheme used.

Proximities. After the user has entered the partitions, using the input program SORTFILE (described below), program PROSORT can be used to perform a computation of proximities between objects. As suggested by Miller (1969), the similarity between two objects a and $b$ is defined as the number of subjects that placed a and $b$ in the same class; subtracting this measure from the total number of subjects yields the corresponding dissimilarity measure. More sophisticated indices were proposed by Rosenberg, Nelson, and Vivekananthan (1968) and by Burton (1972). Empirical tests by Burton (1975) and Drasgow and Jones (1979), however, did not yield compelling evidence in favor of these indices. Furthermore, the present measure lends itself readily to a significance testing procedure, outlined below. As a special feature of PROSORT, proximities may also be computed separately for subgroups of subjects.

Significance of Proximities. With a given number of subjects, some similarities (dissimilarities) will assume large (small) values merely by chance. Therefore, it is desirable to have a statistical procedure for testing the significance of proximity values. The procedure described here was developed by Oldenbürger (1981). It is based on representing each subject's sorting by a Bernoulli distribution. Specifically, a computation is made of the prob-

The author's mailing address is: Fachrichnung Psychologie, Universität des Saarlandes, Postfach, D-6600 Saarbrücken, Federal Republic of Germany. ability $\left(p_{i}\right)$ that two objects randomly drawn from the set of objects under consideration were placed in the same class by Subject $i$. Then, for the sample of $n$ subjects, the distribution of a random variable $X$ is constructed that is the sum of $n$ independent Bernoulli distributed random variables. With $n=2$ subjects, for example, the probability distribution can be constructed as follows: Let $p_{1}$ be the probability that two objects randomly drawn from the object set were sorted together by Subject 1, and let $p_{2}$ be the respective probability for Subject 2 . Assuming statistical independence, the probabilities associated with the $X$-values $\{0,1,2\}$ are: $p(X=0)=\left(1-p_{1}\right)\left(1-p_{2}\right)$, $p(X=1)=p_{1}\left(1-p_{2}\right)+\left(1-p_{1}\right) p_{2}, p(X=2)=p_{1} p_{2}$. Generally, there are $n$ random variables $Y_{i}$ where $p\left(Y_{i}=1\right)$ $=p_{i}$ and $p\left(Y_{i}=0\right)=q_{i}$, with $p_{i}+q_{i}=1$. Then (for $i=1, \ldots, n), X_{n}=\sum_{i} Y_{i}$, with expectation $\mathrm{E}\left(X_{n}\right)=\sum_{i} E\left(Y_{i}\right)$ $=\Sigma_{i} p_{i}$, and variance $\mathrm{V}\left(X_{n}\right)=s_{i} V\left(Y_{i}\right)=s_{i} p_{i} q_{i}$. The associated probabilities can be computed according to the following scheme: $p\left(X_{n}=0\right)=\pi_{i} q_{i}, p\left(X_{n}=k\right)=$ $p\left\{\left[\left(X_{n-1}=k-1\right)\left(Y_{n}=1\right)\right]+\left[\left(X_{n-1}=k\right)\left(Y_{n}=0\right)\right]\right\}$, with $0<k<n, p\left(X_{n}=n\right)=\pi_{i} p_{i}$.

Thus, the construction of the probability distribution makes use of a successive technique that has the advantage of saving much computing time compared with a simultaneous consideration of all $2^{n}$ event combinations. The successive construction method rests on the idea that the distribution of $X_{n}$ can be decomposed into the distributions of $X_{n-1}$ and $Y_{n}$. The program performing such an analysis is called PROSIG. Analogous to PROSORT, this program can also be used with a specified subset of input data (see the input section below).

Correspondence Between Partitions. This kind of analysis is concerned with the problem of measuring the correspondence between object set partitions. Handling sorting data this way can be of interest not only in assessing nominal scale response agreement between two observers or raters, but also in identifying homogeneous subgroups within a sample of subjects in order to differentially analyze and represent proximity data. One of the most often used indices of correspondence is the so-called Rand index (Rand, 1971), which has been rediscovered and/or modified by other researchers (e.g., Brennan \& Light, 1974; Brook \& Stirling, 1984; Fowlkes \& Mallows, 1983; Hubert, 1977; Hubert \& Arabie, 1986). Rand's measure is defined as the ratio of the sum of the number of pairs of objects sorted together in the two partitions being compared (Type A agreements) and the number of pairs of objects sorted in different classes in both partitions (Type B agreements) to the total number of object pairs. A correction for chance agreement, which takes the general form (index value - expected index value)/(1 expected index value), is suggested by Hubert and Arabie (1986). This corrected Rand index is bounded above by 1 (for maximum similarity of both partitions) and takes on the value 0 when it equals its expected value. Both ver- 
sions of this index are implemented by the program RAND. A distance measure proposed by Eckes and Rossbach (1980, pp. 103-105) explicitly weights both Type A and Type $B$ agreements between partitions so that, in contrast to the Rand index, differences based on small classes contribute more to the overall distance than differences based on large classes. The rationale for this weighting scheme is that if the size of Class $C$ is small, the subject has made a relatively fine distinction between the members of $\mathrm{C}$ and all the other members of the object set. It follows that in this case, the average similarity among members of $\mathrm{C}$ is relatively large. Therefore, differences between two partitions based on small classes should be weighted more heavily than those based on large classes that exhibit relatively low internal coherence. The measure takes on values between 0 (identity of two partitions) and 1 (maximum dissimilarity). The name of the associated program is CLUSTIN.

Input. The objects are supposed to be numbered from 1 to $N$, where $N$ is the total number of objects sorted. The input program is SORTFILE. It can handle up to $53 \mathrm{ob}-$ jects. Each partition is entered one after the other in a string. The classes within a partition are to be separated from each other by parentheses, and the objects within classes are to be separated from each other by points. Neither the order of classes within partitions nor the order of objects within classes is of importance. The program checks for possible input errors, such as wrong number of objects or objects entered twice. If an error is detected, the user is informed about the kind of error and requested to re-enter the partition. Correctly entered partitions are subsequently saved on file and (optionally) printed. This file contains the input to all other programs in the package. In order to illustrate the kind of input required, a small example is presented. Suppose three subjects sorted six objects into three classes each (the number of classes need not be equal, of course). The three partitions could look like those shown here:

Partition P1: (1.2.3).(4).(5.6)

Partition P2: (1.2).(3.4.5).(6)

Partition P3: (1.2.3).(4.5).(6)

As mentioned earlier, PROSORT optionally computes object proximities for any subgroup of subjects specified by the user. In this case, the program requests the name of the subgroup and the code numbers of subjects within that group (code numbers may be any subset of numbers $1, \ldots, n)$. The same input information is required by PROSIG if the user wishes to perform a significance test with respect to selected subgroups of subjects.

Output. The output of PROSORT consists of a proximity matrix with similarities above and dissimilarities below the main diagonal (the value 0 is arbitrarily printed in the main diagonal). Using the input data shown above, PROSORT produces the matrix shown in Table 1.

PROSIG yields a tabulated distribution of the random variable $X$. The resulting table shows the probability of randomly drawing a pair of objects sorted in the same class
Table 1

Example of PROSORT Output

\begin{tabular}{llllllll}
\hline & & 1 & 2 & 3 & 4 & 5 & 6 \\
\hline 2 & I & 0 & 3 & 2 & 0 & 0 & 0 \\
3 & I & 0 & 0 & 2 & 0 & 0 & 0 \\
4 & I & 1 & 1 & 0 & 1 & 1 & 0 \\
5 & I & 3 & 3 & 2 & 0 & 2 & 0 \\
6 & I & 3 & 3 & 2 & 1 & 0 & 1 \\
\hline
\end{tabular}

by each of the subjects, the values of the random variable $X$ from 0 to $n$, the probability associated with each of these values, and finally the corresponding distribution function. In addition, the program prints its expectation $\mathrm{E}(X)$ and variance $\mathrm{V}(X)$ as well as an adjusted alpha level $\alpha^{*}=.05 /\left(\begin{array}{l}\mathrm{N} \\ 2\end{array}\right)$ in order to reduce Type I errors when all possible proximities are tested for significance. By applying PROSIG to the three sample partitions, one obtains the results shown in Table 2.

RAND prints a similarity matrix with index values uncorrected for chance above and index values with correction for chance below the main diagonal (the value 0.000 is arbitrarily printed in the main diagonal), as noted in Table 3.

Program CLUSTIN produces a distance matrix with distances based only on Type A agreements above and distances based on both Type A and Type B agreements below the main diagonal (see Table 4).

The effect of the different weighting schemes implied by Rand's (1971) index and the CLUSTIN measure can now be easily demonstrated using the sample input data. Partitions P1 and P13 differ only with respect to Object 5. This difference is based on relatively small classes. Partitions $\mathrm{P} 2$ and $\mathrm{P} 3$ also differ only with respect to one object (Object 3). This time, however, the difference is

Table 2

Example of PROSIG Output

\begin{tabular}{cccc}
\hline$p$ & $k$ & $p(X=k)$ & $p(X \leq k)$ \\
\hline & 0 & 0.3943700 & 0.3943700 \\
.2667 & 1 & 0.4302220 & 0.8245930 \\
.2667 & 2 & 0.1564450 & 0.9810370 \\
.2667 & 3 & 0.0189630 & 1.0000000 \\
\hline $\mathrm{E}(X)=0.8000$ & & & Alpha $=0.0033333$ \\
$\mathrm{~V}(X)=0.5867$ & & & $p(1-$ Alpha $)=0.9966670$
\end{tabular}

Table 3

Example of RAND Output

\begin{tabular}{rrrrc}
\hline & & 1 & 2 & 3 \\
\hline 1 & I & 0.000 & 0.600 & 0.867 \\
2 & I & -0.023 & 0.000 & 0.733 \\
3 & I & 0.659 & 0.318 & 0.000 \\
\hline
\end{tabular}

Table 4

Example of CLUSTIN Output

\begin{tabular}{ccccc}
\hline & & 1 & 2 & 3 \\
\hline 1 & I & 0.000 & 0.667 & 0.500 \\
2 & I & 0.470 & 0.000 & 0.333 \\
3 & I & 0.295 & 0.258 & 0.000 \\
\hline
\end{tabular}


based on relatively large classes. Therefore, the Rand index should yield a larger similarity for P2 and P3 than for P1 and P3; the opposite relationship should hold for the CLUSTIN measure (note that CLUSTIN computes distances, whereas RAND computes similarities). The respective proximities in the output matrices (see Tables 3 and 4) confirm this conjecture.

Requirements. SORTFILE can handle up to 53 objects and requires $5 \mathrm{~KB}$ of storage. The same amount of storage is required by PROSORT. Programs PROSIG and RAND each require $6 \mathrm{~KB}$; CLUSTIN requires $7 \mathrm{~KB}$.

Language. The programs are written in Microsoft BASIC.

Availability. Program listings can be obtained without charge from the author.

\section{REFERENCES}

Boorman, S. A., \& ARABIE, P. (1972). Structural measures and the method of sorting. In R. N. Shepard, A. K. Romney, \& S. B. Nerlove (Eds.), Multidimensional scaling: Theory and applications in the behavioral sciences (Vol. 1, pp. 225-249). New York: Seminar Press.

BRENNAN, R. L., \& LiGHT, R. J. (1974). Measuring agreement when two observers classify people into categories not defined in advance. British Joumal of Mathematical \& Statistical Psychology, 27, 154-163.

Brook, R. J., \& StirLing, W. D. (1984). Agreement between observers when the categories are not specified in advance. British Journal of Mathematical \& Statistical Psychology, 37, 271-282.

BurTon, M. L. (1972). Semantic dimensions of occupation names. In A. K. Romney, R. N. Shepard, S. B. Nerlove (Eds.), Multidimensional scaling: Theory and applications in the behavioral sciences (Vol. 2, pp. 55-71). New York: Seminar Press.

BURTON, M. L. (1975). Dissimilarity measures for unconstrained sorting data. Multivariate Behavioral Research, 10, 402-423.

DRASGow, F., \& Jones, L. E. (1979). Multidimensional scaling of derived dissimilarities. Multivariate Behavioral Research, 14, 227-244.

ECKES, T., \& RosSBACH, H. (1980). Clusteranalysen. Stuttgart: Kohlhammer.

FowlKes, E. B., \& Mallows, C. L. (1983). A method for comparing two hierarchical clusterings. Journal of the American Statistical Association, $78,553-569$.
GRANT, M. J. (1983). AGREE: A BASIC program for assessing similarity in categorization judgments. Behavior Research Methods \& Instrumentation, 15, 611-612.

GREeNBERG, E. A., \& McIsAaC, M. S. (1984). SIMATRIX: A program using the SAS system for multidimensional scaling analysis of sorting data. Behavior Research Methods, Instruments, \& Computers, 16, 407-408.

Hubert, L. J. (1977). Nominal scale response agreement as a generalized correlation. British Journal of Mathematical \& Statistical Psychology, 30, 98-103.

HuberT, L. J., \& ARABIE, P. (1986). Comparing partitions. In W. Gaul \& M. R. Schader (Eds.), Classification as a tool of research (pp. 209215). Amsterdam: North-Holland.

MiLleR, G. A. (1969). A psychological method to investigate verbal concepts. Journal of Mathematical Psychology, 6, 169-191.

OLDENBÜRGER, H. A. (1981). Methodenheuristische aberlegungen und Untersuchungen zur 'Erhebung' und Reprasentation kognitiver Strukturen. Unpublished doctoral dissertation, Göttingen University.

OUD, J. H., \& SATtLer, J. M. (1984). Generalized kappa coefficient: A Microsoft BASIC program. Behavior Research Methods, Instruments, \& Computers, 16, 481.

RAND, W. M. (1971). Objective criteria for the evaluation of clustering methods. Journal of the American Statistical Association, 66, 846-850.

ROSENBERG, S. (1982). The method of sorting in multivariate research with applications selected from cognitive psychology and person perception. In N. Hirschberg \& L. G. Humphreys (Eds.), Multivariate applications in the social sciences (pp. 117-142). Hillsdale, NJ: Erlbaum.

Rosenberg, S., Nelson, C., \& Vivekananthan, P. S. (1968). A multidimensional approach to the structure of personality impressions. Journal of Personality \& Social Psychology, 9, 283-294.

TAKANE, Y. (1981). MDSORT: A special-purpose multidimensional scaling program for sorting data. Behavior Research Methods \& Instrumentation, 13, 698.

TAKANE, Y. (1982). IDSORT: An individual differences multidimensional scaling program for sorting data. Behavior Research Methods \& Instrumentation, 14, 546.

WELDON, D. A., \& BUCHTER, R. (1984). MATGEN: An interactive BASIC program for similarities data matrix generation. Behavior Research Methods, Instruments, \& Computers, 16, 562.

(Manuscript accepted for publication August 1, 1986.) 